Expert Opin Ther Targets. 2015 April ; 19(4): 515-525. doi:10.1517/14728222.2014.987662.

\title{
Novel molecular targets for urothelial carcinoma
}

\author{
Bishoy M. Faltas, MD1, Beerinder S. Karir, MD¹, Scott T. Tagawa, MD¹, and Jonathan E. \\ Rosenberg, MD2 \\ ${ }^{1}$ Division of Hematology and Medical Oncology, Department of Medicine, Weill Cornell Medical \\ College, New York, New York \\ 2Department of Medicine, Memorial Sloan-Kettering Cancer Center and Weill Cornell Medical \\ College, New York, New York
}

\author{
Keywords \\ Urothelial carcinoma; Genomics; Targeted therapy; Molecular targets
}

\section{The need for novel targeted therapies for Urothelial cancer}

Urothelial cancer (UC) affects 75,000 individuals causing the death of 15,000 patients each year in the US alone. There are currently no approved second line therapies for metastatic platinum-resistant urothelial cancer and the median 5-year survival is only $15 \%$. Over the last decade, no new drugs for treatment of UC have been approved by the United States Food and Drug Administration (FDA). The advent of next-generation sequencing technologies is providing an unprecedented insight into the molecular alterations involved in several critical signaling pathways in UC, many of which represent novel therapeutic targets. In addition, new immunotherapeutic approaches are showing promise deserving of further investigation. This review provides an overview of novel molecular targets in UC.

\section{PI3K/AKT/mTOR pathway}

The PI3K-AKT-mTOR signaling pathway regulates many normal cellular processes including cell proliferation, survival, growth, and motility [1]. These cellular processes play important roles in carcinogenesis [2]. Many studies over the last decade have identified frequent alterations in various components of this pathway. These include the catalytic subunit-PIK3CA, the negative regulator-PTEN, the downstream kinase-AKT and the negative regulator of mTOR-TSC[2]. Over the last decade, multiple molecular alterations in this pathway have been implicated in many different cancers [3][4][5][6]. A recent analysis of driver cancer genes across various tumor types identified significant alterations in PI3KAKT-mTORsignaling components especially PIK3CA in bladder, endometrial, breast, lung and head \& neck cancers. [7].

Recent genomic analyses of two large cohorts of UC patients showed frequent alterations in PIK3-AKT-mTOR pathway. The largest analysis from the Cancer Genome Atlas (TCGA) 
network, which included a cohort of 131 chemotherapy-naïve high grade muscle invasive UC patients (T2-T4a, Nx, Mx), reported mutations and copy number alterations (CNAs) within this pathway in $42 \%$ of tumors [8]. Another study of potentially actionable genetic aberrations in bladder cancer also found a similar prevalence of alterations in $30 \%$ of the patients. This study included 97 high-grade UC patients with $35 \%$ of patients having received neo-adjuvant chemotherapy, and may account for some of the differences observed between the two cohorts [10].

\subsection{PIK3CA (Phosphatidylinositol-4,5-bisphosphate 3-kinase, Catalytic subunit Alpha)}

Phosphatidylinositol 3-kinase is composed of regulatory subunit and a catalytic subunit. The catalytic subunit phosphorylates phosphatidylinositol, phosphatidylinositol 4-phosphate and phosphatidylinositol 4,5-bisphosphate to generate PIP3 (phosphatidylinositol 3,4,5triphosphate). PIP3 is responsible for recruiting proteins like RAC-alpha serine/threonineprotein kinase (AKT1) and 3-phosphoinositide dependent protein kinase-1(PDPK1) to the membrane and activating signaling cascades involved in cell growth, survival and motility[10]. Several mutations in the catalytic domain have been uncovered [11]. The TCGA analysis of non-metastatic bladder cancer patients found PIK3CA activating point mutations in $20 \%$ of the tumors, mainly in a hotspot area in the helical domain near E545 [8]. This leads to an amino acid substitution at position 545 (glutamic acid $\{\mathrm{E}\}$ to a lysine $\{\mathrm{K}\})$ in PIK3CA, thereby increasing catalytic activity and downstream signaling. Previous analyses had also found similar frequencies and the same spectrum of PIK3CA mutations in bladder cancer[11]. A study by Platt et al. assessing the frequency of mutations in PIK3CA pathway in bladder cancer, reported 52\% mutations in E545k and 13\% mutations in H1047R compared to $33 \%$ and $46 \%$ respectively in non-bladder cancers according to COSMIC study. Therefore, the frequency of PIK3CAmutation at these mutational hotspots PIK3CA observed in UC differs from other tumor types[11]. These mutations confer selective growth advantage and may contribute to intraepithelial cell movement within the urothelium [12]. PIK3CA alterations also are suggested to have a prognostic significance. They co-occur with FGFR3 mutations in $26 \%$ of papillary urothelial neoplasms of uncertain malignant potential[13]. Tumors bearing PIK3CA mutations tend to have lower recurrence rates whereas FGFR3 mutant PIK3CA wild type tumors have higher recurrence rates[14]. Mutations in PIK3R1, which is a negative regulator of PIK3CA, have also been reported in UC, although the functional implications of these mutations are unclear at this time. [15]

Early phase clinical trials of PI3K pathway inhibitors are currently underway in UC. A phase II clinical trial testing buparlisib, a pan-class I selective phosphoinositide 3-kinase (PI3K) inhibitor, in UC isongoing (NCT01551030). The trial is enrolling metastatic transitional cell UC patients with alterations in the PI3K pathway [16]. Preliminary results from another phase I study of PI3K inhibitor GSK2126458 in 170 patients with advanced solid cancer have shown objective responses ( 1 of 3 UC patients bearing PIK3CA mutations), although responses were also observed in wild-type patients ( 2 of $15 \mathrm{UC}$ patients) [17]. Further work is required to characterize the relationship between PIK3CA mutation and response to PI3-kinase inhibition in UC. 


\subsection{PTEN (Phosphatase and Tensin Homolog)}

PTEN is a tumor suppressor gene implicated in several cancers. Loss of PTEN activity has been shown to induce urothelial hyperplasia/carcinoma and increased invasiveness of bladder cancer cell lines[18][19]. In an analysis of 69 primary and 23 recurrent tumor samples of both non-muscle invasive and Muscle Invasive Bladder Cancer (MIBC) using array comparative genomic hybridization, loss of heterozygosity of the PTEN region was reported in $12 \%$ and homozygous deletion was reported in $1.4 \%$ of tumors. Reduced PTEN protein expression was confirmed using immunoblotting in $49 \%$ of tumors [20]. The TCGA analysis of urothelial cancer has found PTEN CNAs in $13 \%$ of bladder tumors. Recently, PTEN loss has also been shown to lead to PI3K inhibitor resistance in other cellular contexts[21]; it was reported that PI3K inhibitor refractory lesions in a breast cancer patient had PTEN loss whereas responding lesions had no PTEN alterations.

\subsection{AKT (RAC-alpha serine/threonine-protein kinase)}

AKT is a regulator of the PI3K pathway, which gets phosphorylated to influence a wide range of cellular processes. It has been proposed that AKT activation is not directly related to PTEN loss or PIK3CA-activating mutations and therefore alternative activating AKT mutations are expected to be discovered [22]. AKT1 (E17K) mutations have been reported in $2.7 \%$ of bladder tumors[23]. This mutation results in constitutive activation of AKT and its recruitment to the plasma membrane without the signaling of PIK3 kinase. Also, it has been shown to co-operate with other known AKT1 activating mutation[23]. The TCGA analysis also reported AKT1 alterations (amplification and homozygous deletion) in 6\%, AKT1 mutation in 2\%, and AKT3 overexpression in $10 \%$ of the samples independent of copy number[8].

\subsection{TSC (Tuberous Sclerosis Complex)}

TSC is a negative regulator of mTOR. Several studies have shown a variable incidence of TSC mutation ranging between 8-16\%[20],[15][8]. A recent genomic analysis of 35 stage IV UC's identified TSC1 mutations in 6\% of the cohort [25]. Most of the mutations in the bladder cancer cohort of TCGA were found to be truncating, resulting in loss of function. Preclinical studies have shown that in TSC1 mutant bladder cancers, combined mTOR/ hsp90 inhibition is a promising strategy[25]. TSC mutations are also shown to be a marker of sensitivity to mTOR inhibition. Whole genome sequencing in a patient who showed near complete response to everolimus in a phase II study revealed a 2 base pair deletion (nonsense mutation) in the TSC1 gene. Subsequent genomic analysis of other patients on the same trial showed that everolimus responders carried more TSC1 mutations (non-sense or missense) as compared to non-responders who had wild type TSC1 [26].

\section{5 mTOR (mammalian Target of Rapamycin)}

mTOR plays a central role in metabolism, cell proliferation and growth, and inhibiting this proteinis an important target for therapeutic intervention [27]. However, the results of two phase IIclinical trials of mTOR inhibitors as single agent therapy in UC patients haven't been promising in unselected patients [28][29]. In a phase II single arm, non-randomized study in 45 UC patients who had progressed on cytotoxic agents, one patient had near 
complete response, one had a partial response, and several had minor responses. The primary end point of 2-month PFS in more than 70\% of patients was not achieved[28]. Subsequent whole genome sequencing of the patient who showed near complete response to everolimus in the study had non-synonymous mutations in TSC, lending biomarker credibility to this regulator of mTOR (as noted above)[26]. Analysis of another phase II study of everolimus in platinum-resistant patients led to conclusion that intact PTEN activity was required for mTOR inhibitor sensitivity due to feedback through AKT[29][30]. Recently, a phase I study of mTOR inhibitor in combination with pazopanib has been reported in which a patient who showed 14 month complete response to therapy had two activating mutations in mTOR[31]. These findings highlight the importance of biomarkerenriched trial designs in UC targeted therapy trials.

\section{HER2 pathway}

Human Epidermal growth factor Receptor 2 (HER2) is a receptor tyrosine kinase that dimerizes with other ligand-bound receptors to form a heterodimer, thereby activating downstream signaling through MAPK and PI3K pathways. It is encoded by the ERBB2 gene on chromosome 17 and alterations in this gene have been reported in various cancers, most notably breast cancer (14.9\%) and uterine tumors (10.4\%)[32][6]. Earlier studies reported overexpression of this gene in 5\% of urothelial tumors[33], although the frequency varies in different studied cohorts[34][35][36]. According to recent TCGA data, molecular alterations of ERBB2(HER2) gene have been found in 13\% of UC [8] with overexpression in $7 \%$ suggesting a potential role of HER2 targeted therapy in selected UC.

Approved therapies targeted to Her2, such as trastuzumab and lapatinib for breast cancer, have already been under investigation for treatment of HER2-amplified UC[37]. In a single arm phase II study, trastuzumab was combined with carboplatin, gemcitabine and paclitaxel in 44 patients with HER2 overexpression as measured by immunohistochemistry, FISH, or serum Her2 protein levels. Objective response was seen in $70 \%$ of patients with $11 \%$ showing complete response and 59\% experiencing partial responses. The combination was well tolerated with low-grade cardiac toxicity seen in $22.7 \%$ patients. The authors concluded that the demonstrated safety and efficacy of combination warranted a randomized trial for understanding the efficacy of trastuzumab in HER2 positive UC patients[38]. No conclusions could be obtained from another phase II study of platinum-based chemotherapy with or without trastuzumab as it failed to accrue the required number of patients to achieve adequate statistical power[39]. In another ongoing phase II study, ERBB2 (HER2) is targeted by cellular autologous immunotherapy in adjuvant setting of high-risk HER2 positive UC patients[40]. Interim results reported in 13 patients on this study has found DN24-02 to be safe and out of 10 patients who completed all infusions, 7 patients have shown favorable immune responses [41]. Lapatinib has also been tested as maintenance therapy following first-line chemotherapy in HER2 or EGFR overexpressing UC patient in a fully accrued phase II/III study; results are awaited[42].

A recent study using Next Generation Sequencing (NGS) detected activating mutations in the extracellular domain of ERBB in micropapillary urothelial carcinoma which had tested negative for overexpression by immunohistochemistry (IHC)[43]. TCGA bladder study has 
also identified recurrent missense mutations in ERBB2 and ERBB3 (11\%) genes. The frequency of ERBB2 mutations in the bladder cohort of TCGA was in fact more than identified in the breast cancer TCGA cohort [8]. It has already been shown in inflammatory breast cancer patients that lapatinib is effective for ERBB2 mutated, non amplified tumors [44]. Based on the same concept, a phase II trial testing Neratinib, a dual EFGR and HER2 inhibitor, is recruiting HER2 mutated solid tumor patients and includes a bladder cancer cohort [45].

\section{EGFR/HER1 pathway}

Epidermal Growth Factor Receptor (EGFR) is activated when the ligand binds to its extracellular ligand-binding domain resulting in receptor dimerization and autophosphorylation by the intracellular kinase domain. Following activation, a downstream signaling cascade is initiated that drives many cellular responses including cell proliferation and resistance to apoptosis [46], which are two of the hallmarks of cancer [47].

ERBB1 (EGFR) is frequently over expressed in urothelial carcinomas, and TCGAdata reported amplification in $11 \%$ of 131 samples, with $9 \%$ potentially responsive to anti-EGFR therapy. Various EGFR inhibitors/antibodies have been tested in several clinical trials with only limited efficacy. A case report in a 62 year old female patient with urothelial carcinoma of kidney harboring rare EGFR double mutation showed good clinical response to antiEGFR inhibitor gefitinib[48]. In a phase II study of gefitinib in cohort of 31 patients with metastatic or locally advanced UC, screened for EGFR expression by IHC, median progression free survival was 2 months with only two patients surviving past 6 months without disease progression and only one patient showing confirmed partial response[49].

Anti-EFGR therapies have also been tested in combination with chemotherapeutic agents. A phase II randomized non-comparative study was conducted to determine the efficacy of cetuximab with or without paclitaxel in 39 patients with previously treated UC. Cetuximab monotherapy arm showed no benefit with nine out of the eleven patients progressing by 8 weeks thereby leading to closure of the monotherapy arm. The combination arm (cetuximab plus paclitaxel) showed a median progression free survival of 16.4weeks (95\% CI, 12 to 25.1 weeks)[50]. In another phase II trial, geftinib was added to cisplatin and gemcitabine in patients with advanced UC. No improvement in response rates or survival with the addition of geftinib was observed in comparison to historical controls receiving cisplatin and gemcitabine [51]. Both trials did not require the presence of EGFR alterations as a criterion for enrollment. These trials suggest that in unselected patients, urothelial tumors are generally resistant to anti-EFGR therapies.

Tissue profiling and analysis of cells resistant to anti-EGFR therapy identified potential the induction of epithelial to mesenchymal transition (EMT) as a resistance mechanism[52]. Administration of HDAC inhibitors in urothelial cancer cell lines restored sensitivity to EGFR inhibitors in resistant cells by reversing the EMT process through the induction of 'mesenchymal to epithelial transition' [52]. 


\section{FGFR3 pathway}

FGFR3 belongs to receptor tyrosine kinase family of Fibroblast Growth Factor Receptor (FGFR), having members which differ in ligand affinities and tissue distribution. FGFR3 plays an essential role in the regulation of cell proliferation, differentiation and apoptosis. This receptor tyrosine kinase interacts with fibroblast growth factors leading to downstream signals thereby affecting mitogenesis and differentiation [53]. Upregulation of FGFR expression leads to cell transformation and cancer. Somatic mutations in this gene occur in bladder cancer, multiple myeloma and T-cell lymphoma.

FGFR3 was found to be mutated with a high frequency in up to $84 \%$ of pTa tumors and $21 \%$ of T1 tumors [54][55]. As compared to non-muscle invasive tumors, genomic analysis in 35 stage IV UC's revealed FGFR3 alterations in only $11 \%$ tumors [25]. Another analysis done in 97 high grade UC samples(containing 85\% muscle-invasive tumors)using array comparative genomic hybridization (for CNA's), mass spectroscopy-based genotyping and Sanger sequencing (for mutation profiling), identified FGFR3 molecular alterations in $15.5 \%$ samples with mutations in $13.4 \%$ of the cases and amplification in $1 \%$ [56]. The TCGA analysis also identified significant alterations in the FGFR3 gene. Out of 127 muscle invasive UC samples, FGFR3 was altered in $19 \%$ of cases, with mutations in $12.6 \%$, overexpression in $3.1 \%$, fusions in $2.4 \%$ and deletion in $0.8 \%$ [8].

In addition to mutations, chromosomal translocation is another mechanism of FGFR3 activation in bladder cancer. FGFR3-TACC3 (Transforming Acid Coiled Coil 3) activating fusions have been identified in urothelial cancer leading to increased activation of MAPK pathway [57]. As mentioned earlier, the TCGA analysis also identified this chromosomal rearrangement in three UC samples. The breakpoints were located in introns 16 or 17 for FGFR3 and in intron 10 of TACC3. The resulting fusion protein includes the aminoterminal 758 amino acids of FGFR3 fused with the carboxy-terminal 191 amino acids of TACC3 hypothesized to auto-dimerize and activate the FGFR3 kinase domain [8]. These chromosomal translocations are potential therapeutic targets for FGFR3-directed therapy.

FGFR3 mutations are attractive therapeutic targets for both muscle invasive and superficial UC in preclinical models. FGFR inhibition has cytostatic and cytotoxic effects in FGFRdependent bladder cancer cells[58].A study of three small molecule FGFR3 inhibitors (PD173074, TKI-258 and SU5402) tested on a panel of 13 bladder cancer cell lines demonstrated a correlation between response and FGFR3 expression levels in each cell line[59]. Based on these results, clinical trials of FGFR3-directed therapy were designed. There is an ongoing phase II trial of dovitinib, a small molecule inhibitor which inhibits FGFR3 in histologically confirmed non muscle invasive (Ta, T1,Tis stage) bladder cancer harboring FGFR mutation or overexpression that is refractory to BCG therapy [60]. A phase II clinical study of dovitinib, was terminated because of insufficient accrual [61].Nextgeneration FGFR inhibitors are currently undergoing development. A phase I study testing selective pan FGFR inhibitor, BGJ398, was found to be safe and showed clinical activity in solid tumors with FGFR genetic alteration especially UC[62]. Similarly another pan FGFR inhibitor, JNJ-42756493, has been shown to have a tolerable safety profile and anti-tumor activity with one of the patient harboring FGFR3-TACC3 translocation showing confirmed 
PR [63] suggesting that patients withFGFR3 fusions may be more sensitive to these newer inhibitors.

\section{EGFR, HER2 and FGFR cross talk: Rationale for combined blockade}

There is a significant level of cross-talk and redundancy among different signaling pathways resulting in both inherent and acquired resistance to targeted therapies [64]. Inhibiting one pathway usually leads to a compensatory activation of other cellular pathways. For instance: FGFR mutant cancer cell lines resistant to anti-FGFR therapy activate EGFR signaling as a compensatory mechanism[65]. This concept provides a rationale for using targeted therapy combinations of inhibitors that target different pathways to overcome such resistance mechanisms.

Small molecule inhibitors of tyrosine kinases that can concurrently block multiple receptors provide another approach to target different pathways with a single drug. Cabozantinib, an inhibitor of c-MET and VEGFR2, is being tested in phase II study of relapsed or refractory metastatic UC and preliminary results have demonstrated clinical activity[66].

\section{HSP (Heat Shock Protein)}

Heat shock proteins (HSP) are essential for cell survival during physiological and environmental stress acting as molecular chaperones and by preventing the aggregation of caspases thus preventing cell death. HSPs are involved in tumor progression and the development of treatment resistance through their cytoprotective activity.

Various members of HSP are expressed in a wide range of cancers including UC. HSP27 is encoded by HSBP1 gene and reported in the TCGA analysis to be amplified in 1.6\% of samples HSP27 knockdown inhibits bladder tumor growth, and has shown evidence of enhanced antitumor activity with taxane therapy [67]. The silencing of HSP27 in UC has been achieved by targeting its mRNA by antisense oligonucleotide- OGX-427. A phase II study for evaluating the safety and efficacy of OGX-427 in combination with gemcitabine and cisplatin in advanced transitional carcinoma has completed accrual[68]. Another ongoing study is testing OGX-427 with docetaxel in UC patients who had received a prior platinum containing regimen [69].

Another member of this group of proteins, HSP 70-2 expression is also associated with early spread and progression of urothelial carcinoma of bladder and inhibition of HSP 70-2 significantly suppressed the progression of tumor in an in vivo mouse xenograft study [70].

\section{Chromatin remodeling}

Chromatin remodeling is a process by which chromatin structure is modified in order to expose the condensed genomic DNA to regulatory transcription proteins. This dynamic change in chromatin structure is essential for controlling gene expression. Chromatin remodeling occurs through enzymatic histone modification or ATP dependent complexes which restructure the nucleosomes[71]. 
Alterations in the chromatin regulatory machinery are frequently reported in UC. In a genomic analyses of 97 patients, 59\% had mutations in genes associated with chromatin remodeling[72]. This was confirmed by the TCGA study of 131 bladder carcinoma patients, which showed mutations/CNAs of histone modifying genes in $89 \%$ of samples. Most notably the CREBBP gene which encodes a histone acetyltransferase demonstrated mutations in $12 \%$ and CNAs in $14 \%$ of cases. The methyltransferaseMLL2 showed a mutation rate of $27 \%$ and a $3 \%$ CNAs rate, Similarly, KDM6A which encodes for a demethylating protein showed mutations in $24 \%$ and CNAs in $3 \%$ of cases. The majority of these molecular alterations were inactivating resulting in loss of function. The components of SWI/SNF nucleosomes complex were altered in 64\% samples with the most frequent alterations seen in ARID1A (25\% mutations and 3\% CNAs) [8]

Targeting epigenetic regulation in UC can be achieved using DNA methyltransferase inhibitors(DNMTi) and histone deacetylase inhibitors (HDACi). A phase I trial of decitabine (DNMTi) including bladder cancer patients to determine the safety and the maximum tolerated dose is completed. However the results are not available yet[73]. Changes in HDAC expression have been studied in UC tissues and cell lines and pharmacologically targeted. Upregulation of HDAC2 and HDAC 8 and downregulation of HDAC 4, HDAC5 and HDAC7 mRNA are common finding but not predictive of treatment response. Vorinostat (SAHA-suberanilohydroxamic acid), a pan HDACi,has been shown to induce G2/M arrest, increased sub-G1 fraction, upregulated p21 and down regulated TS in UC[74]. A phase I study of intravenous SAHA in advanced tumors including UC showed good antitumor activity with two UC patients experiencing objective tumor regression [75]. Tolerable safety profile and antitumor activity has also been observed in another phase I trial with orally administered SAHA in advanced tumors [76]. A phase II clinical trial of vorinostat in locally recurrent or metastatic UC patients proved this drug to be highly toxic causing predominantly cytopenias and thrombocytopenic bleeding. Also, it had limited efficacy in patients with advanced TCC[77]. The adverse side effect profile of pan HDACi emphasized the need for developing specific HDACi. A preclinical study done to evaluate HDAC6 as a potential target, using HDA6 specific inhibitor tubacin has also not proved to be a promising target for treatment of UC[78]. Treatment of histone acetyl-transferase mutated bladder cancers with HDACi's represents an appealing approach. A new clinical trial testing mocetinostat, a selective HDACi, is enrolling patients with EP300 and CREBBP inactivating mutations (http://clinicaltrials.gov/show/NCT02236195).

\section{Immunotherapy}

Resistance to cell death and evasion of immune destruction are known cancer hallmarks[79]. Progress in immunotherapy clinical trials across various tumor types promises to deliver significant benefit to patients with several solid tumor types including UC [80] [81].

In non-muscle invasive UC, immunotherapy in the form of intravesical BCG (bacillus Calmette-Guérin) has been demonstrated to have benefit in multiple clinical trials and is a standard recommendation since many years[82]. The role of interferon alpha alone and in combination with BCG has also been tested [83]. Newer immunotherapeutics are being investigated in multiple settings including ipilimumab, a monoclonal antibody against 
CTLA-4(Cytotoxic T-Lymphocyte Antigen 4), which is already approved by the FDA for treatment of advanced melanoma. As a monotherapy in the neoadjuvant setting, a trial was performed to evaluate the safety of ipilimumab and immune monitoring in 12 patients with localized urothelial carcinoma prior to cystectomy. Two of 6 patients in the high dose cohort had to delay surgery due to immune-related Adverse Events (AE's), though all patients recovered and toxicity was generally limited to grade 1-2. Immune correlates were associated with overall survival in a companion study in patients with melanoma[84]. Another phase II study is evaluating the addition of immunotherapy to chemotherapy by using gemcitabine, cisplatin and ipilimumab as the first line drugs in the treatment of urothelial carcinoma is currently recruiting patients. In this study total of six cycles of chemotherapy are planned with ipilimumab to be added from third cycle onwards[85].

Programmed cell death protein 1 (PD-1) and its ligand are attractive targets of cancer immunotherapeutics as they facilitate tumor cell death. Recent phase 1 clinical study data suggests that immune checkpoint blockade has activity in patients with previously treated metastatic UC. In this study MPDL3280A (anti PD-L1) was found to be safe and half of the patients had rapid ongoing responses [86]. The FDA has already granted Breakthrough Therapy Designation to MPDL3280A in bladder cancer. A phase II trial designed to evaluate the effect of same anti-PDL1 drug in patients with locally advanced or metastatic UC is ongoing and has finished recruiting platinum-refractory subjects. This single arm study enrolled patients in one of the two cohorts: treatment naïve and patients who are ineligible for platinum-containing therapy, and patients who have progressed during or following a prior platinum based chemotherapy regimen. Tissue specimens are required to be submitted for biomarker analysis, but prospective identification of PDL1 expression is not required[87]. Further studies comparing MPDL3280A with vinflunine and other second line treatments are planned. Evaluation of immune checkpoint blockade in different bladder cancer disease states is likely to yield significant therapeutic advances in coming years.

Lenalidomide is an immunomodulatory drug and has been approved by FDA for multiple myeloma and myelodysplastic syndromes. Its activity in solid tumors including UC is being tested in numerous clinical trials. In an ongoing phase II study, efficacy of lenalinomide and intravesical BCG in bladder cancer patients will be assessed using progression free survival as a measure of primary outcome. This study is still recruiting participants[88].

\section{Molecular mechanisms of cisplatin resistance in urothelial cancer}

Cisplatin induces inter- and intra strand DNA crosslinks resulting in apoptotic cell death [89][90]. UC cell lines treated with gradually increasing doses of ciplsatin develop resistance [91], while these in vitro models are useful for study molecular study resistance mechanisms they do not necessarily replicate the biology of platinum-resistance in patients or the role of the microenvironment. Resistance to cisplatin is complex, multilayered phenomenon that is not completely understood [92]. Resistance mechanisms can be generally classified into those that decrease the intracellular concentration of cisplatin and those that alter the DNA damage response or promote survival in the face of DNA damage [92]. Transporters such as The Multidrug resistance protein (MRP) encoded by the ABCC gene or P-glycoprotein encoded by MDR1 gene are not consistently involved in UC 
resistance to cisplatin [93][94][95][96][97]. On the other hand, another transporter; the calanicular multispecific organic anion transporter (cMOAT/MRP2) has been shown to be elevated in platinum-resistant cancer cells derived from T24 cells [98] as well as in tumors from patients treated with MVAC [99]. Evidence suggests that detoxification of cisplatin by the intracellular thiols glutathione and thioredoxin play an important role in mediating resistance [100][101][102]. DNA repair of cisplatin-induced DNA damage occurs through various pathways including the nucleotide excision repair, homologous recombination and non-homologous end joining repair [92]. Somatic mutations in ERCC2, a gene involved in the nucleotide excision-repair gene mutations were present in $38.5 \%$ of samples from patients responding to Cisplatin neoadjuvant chemotherapy for UC compared to $0 \%$ of nonresponder samples [103]. An ongoing clinical trial is evaluating the correlation of carboplatin-DNA monoadduct formation and ERCC1 expression with clinical responses to carboplatin-based chemotherapy [104]

Overexpression of anti-apoptotic proteins such as BCL-2 has been associated with platinum resistance in UC cell lines, siRNA knockdown restored platinum sensitivity [105][106]. In another study, restoring BCL-2 expression decreases the sensitivity of xenografts to cisplatin treatment[107]. Epigenetic changes such as DNA methylation have been implicated in platinum-resistance; the histone deacetylase inhibitor trichostatin A has been demonstrated to sensitize UCcells to cisplatin[108].

Cisplatin resistance in UC is a complex cellular process that involves several pathways. These mechanisms are not completely understood leading to the persistence of platinumresistance in the clinical setting.

\section{Expert opinion}

UC continues to be a lethal disease with no effective second-line therapies. In contrast to other tumors, no novel agents have been FDA-approved over the last two decades and chemotherapy continues to be the mainstay of treatment. The advent of next generation high throughput sequencing has led to novel insights into the molecular underpinnings of UC. The recent TCGA analysis provides a comprehensive analysis of primary UC and has identified several recurrent molecular alterations. Some of these alterations are already validated molecular targets in UC and other cancer types. For others, functional studies of the impact of these alterations on the biology of the disease are still needed. Platinumresistance in UC continues to be a daunting clinical problems and the underlying molecular mechanisms remain poorly understood. Understanding the genomic landscape of platinumresistant and metastatic UC could be a starting point for understanding this important phenomenon.

Clinical trials evaluating targeted therapeutic strategies are ongoing but success has been limited to date. Conducting genomics-driven clinical trials that enroll patients based on a distinct molecular subtype is challenging, but offers an opportunity to improve clinical outcomes for patients with UC. Genomic profiling of tumors from response outliers in targeted clinical trials will also provide us with significant insights into the biology of the disease not only in extreme-responders but also providing leads to key biological processes 
in other patients. Immunotherapy is a promising treatment approach and its use in UC is expected to expand in the next few years as PD-1 and PD-L1 inhibitors clinical trials.

Although drugs altering the bladder cancer epigenome have not yet shown clinical benefit, improvement in drug and clinical trial design may lead to improved clinical trial outcomes.

In conclusion, next-generation sequencing technologies are providing unprecedented insight into the molecular landscape of UC and revealing several molecular targets. Several targeted therapy trials are currently underway, paving the way for precision medicine of bladder cancer patients.

\section{References}

1**. Luo J, Manning BD, Cantley LC. Targeting the PI3K-Akt pathway in human cancer: rationale and promise. Cancer Cell. 2003; 4(4):257-62.**Landmark paper linking PI-3-K pathway with cancer. [PubMed: 14585353]

2. Yuan TL, Cantley LC. PI3K pathway alterations in cancer: variations on a theme. Oncogene. 2008; 27(41):5497-510. [PubMed: 18794884]

3. The Cancer Genome Atlas Research Network. Comprehensive genomic characterization defines human glioblastoma genes and core pathways. Nature. 2008; 455(7216):1061-8. [PubMed: 18772890]

4. The Cancer Genome Atlas Research Network. Integrated genomic analyses of ovarian carcinoma. Nature. 2011; 474(7353):609-15. [PubMed: 21720365]

5. The Cancer Genome Atlas Research Network. Comprehensive molecular characterization of clear cell renal cell carcinoma. Nature. 2013; 499(7456):43-9. [PubMed: 23792563]

6. The Cancer Genome Atlas Research Network. Integrated genomic characterization of endometrial carcinoma. Nature. 2013; 497(7447):67-73. [PubMed: 23636398]

$7 * *$. Lawrence MS, Stojanov P, Mermel CH, et al. Discovery and saturation analysis of cancer genes across 21 tumour types. Nature. 2014; 505(7484):495-501. **Paper analyzing the TCGA genomic data across various cancer types. [PubMed: 24390350]

8**. The Cancer Genome Atlas Research Network. Comprehensive molecular characterization of urothelial bladder carcinoma. Nature. 2014; 507(7492):315-22. **TCGA analysis of Urothelial tumors. [PubMed: 24476821]

9. Iyer G, Al-Ahmadie H, Schultz N, et al. Prevalence and co-occurrence of actionable genomic alterations in high-grade bladder cancer. J Clin Oncol. 2013; 31(25):3133-40. [PubMed: 23897969]

10. PIK3CA phosphatidylinositol-4,5-bisphosphate 3-kinase, catalytic subunit alpha [Homo sapiens (human)] - Gene - NCBI [Internet]. Available from: http://www.ncbi.nlm.nih.gov/gene? cmd=Retrieve \&dopt=full_report\&list_uids=5290

11. Platt FM, Hurst CD, Taylor CF, et al. Spectrum of phosphatidylinositol 3-kinase pathway gene alterations in bladder cancer. Clin Cancer Res. 2009; 15(19):6008-17. [PubMed: 19789314]

12. Ross RL, Askham JM, Knowles MA. PIK3CA mutation spectrum in urothelial carcinoma reflects cell context-dependent signaling and phenotypic outputs. Oncogene. 2013; 32(6):768-76. [PubMed: 22430209]

13. López-Knowles E, Hernández S, Malats N, et al. PIK3CA mutations are an early genetic alteration associated with FGFR3 mutations in superficial papillary bladder tumors. Cancer Res. 2006; 66(15):7401-4. [PubMed: 16885334]

14. Dueñas M, Martínez-Fernández M, García-Escudero R, et al. PIK3CA gene alterations in bladder cancer are frequent and associate with reduced recurrence in non-muscle invasive tumors. Mol Carcinog. 2013 Oct.:1-11.

15. Sjödahl G, Lauss M, Gudjonsson S, et al. A systematic study of gene mutations in urothelial carcinoma; inactivating mutations in TSC2 and PIK3R1. PLoS One. 2011; 6(4):e18583. [PubMed: 21533174] 
16. Buparlisib in Metastatic Transitional Cell Carcinoma of the Urothelium - Full Text View ClinicalTrials.gov [Internet]. Available from: http://clinicaltrials.gov/show/NCT01551030

17. PI3K kinase inhibitor GSK2126458 (GSK458): clinical activity in select patient (pt) populations defined by predictive markers (study P3K112826) [Internet]. Available from: http:// abstracts.webges.com/viewing/view.php? congress=esmo2012\&congress_id=370\&publication_id=1500

18. Tsuruta H, Kishimoto H, Sasaki T, et al. Hyperplasia and carcinomas in Pten-deficient mice and reduced PTEN protein in human bladder cancer patients. Cancer Res. 2006; 66(17):8389-96. [PubMed: 16951148]

19. Puzio-Kuter AM, Castillo-Martin M, Kinkade CW, et al. Inactivation of p53 and Pten promotes invasive bladder cancer. Genes Dev. 2009; 23(6):675-80. [PubMed: 19261747]

20. Platt FM, Hurst CD, Taylor CF, et al. Spectrum of phosphatidylinositol 3-kinase pathway gene alterations in bladder cancer. Clin Cancer Res. 2009; 15(19):6008-17. [PubMed: 19789314]

21. Loss of PTEN leads to clinical resistance to the PI3Ka inhibitor BYL719 and provides evidence of convergent evolution under selective therapeutic pressure [Internet]. Available from: http:// www.abstractsonline.com/Plan/ViewAbstract.aspx?sKey=1ec08f7b-2c2b-44b4a03a-5fea49231668\&cKey $=6 a f 1 c b 5 f-9156-4 d 86-b f 0 c-2 c 45 c 9 a e 0324 \& m K e y=\{6$ FFE1446A164-476A-92E7-C26446874D93\}

22. Calderaro J, Rebouissou S, de Koning L, et al. PI3K/AKT pathway activation in bladder carcinogenesis. Int J Cancer. 2013; 3:1776-1784. [PubMed: 24122582]

23. Askham JM, Platt F, Chambers PA, et al. AKT1 mutations in bladder cancer: identification of a novel oncogenic mutation that can co-operate with E17K. Oncogene. 2010; 29(1):150-5. [PubMed: 19802009]

24. Ross JS, Wang K, Al-Rohil RN, et al. Advanced urothelial carcinoma: next-generation sequencing reveals diverse genomic alterations and targets of therapy. Mod Pathol. 2014; 27(2):271-80. [PubMed: 23887298]

25. Guo Y, Chekaluk Y, Zhang J, et al. TSC1 involvement in bladder cancer: diverse effects and therapeutic implications. J Pathol. 2013; 230(1):17-27. [PubMed: 23401075]

26**. Iyer G, Hanrahan AJ, Milowsky MI, et al. Genome sequencing identifies a basis for everolimus sensitivity. Science. 2012; 338(6104):221. **Seminal paper showing genomic basis for mTOR inhibitor response. [PubMed: 22923433]

27. Gomez-Pinillos A, Ferrari AC. mTOR signaling pathway and mTOR inhibitors in cancer therapy. Hematol Oncol Clin North Am. 2012; 26(3):483-505. [PubMed: 22520976]

28. Milowsky MI, Iyer G, Regazzi AM, et al. Phase II study of everolimus in metastatic urothelial cancer. BJU Int. 2013; 112(4):462-70. [PubMed: 23551593]

29. Seront E, Rottey S, Sautois B, et al. Phase II study of everolimus in patients with locally advanced or metastatic transitional cell carcinoma of the urothelial tract: clinical activity, molecular response, and biomarkers. Ann Oncol. 2012; 23(10):2663-70. [PubMed: 22473592]

30. Seront E, Pinto A, Bouzin C, et al. PTEN deficiency is associated with reduced sensitivity to mTOR inhibitor in human bladder cancer through the unhampered feedback loop driving PI3K/Akt activation. Br J Cancer. 2013; 109(6):1586-92. [PubMed: 23989949]

31. Wagle N, Grabiner BC, Van Allen EM, et al. Activating mTOR Mutations in a Patient with an Extraordinary Response on a Phase I Trial of Everolimus and Pazopanib. Cancer Discov. 2014; 4(5):546-53. [PubMed: 24625776]

32. The Cancer Genome Atlas Research Network. Comprehensive molecular portraits of human breast tumours. Nature. 2012; 490(7418):61-70. [PubMed: 23000897]

33. Laé M, Couturier J, Oudard S, et al. Assessing HER2 gene amplification as a potential target for therapy in invasive urothelial bladder cancer with a standardized methodology: results in 1005 patients. Ann Oncol. 2010; 21(4):815-9. [PubMed: 19889613]

34. Krüger S, Weitsch G, Büttner H, et al. HER2 overexpression in muscle-invasive urothelial carcinoma of the bladder: prognostic implications. Int J Cancer. 2002; 102(5):514-8. [PubMed: 12432555] 
35. Latif Z, Watters AD, Dunn I, Grigor KM, et al. HER2/neu overexpression in the development of muscle-invasive transitional cell carcinoma of the bladder. Br J Cancer. 2003; 89(7):1305-9. [PubMed: 14520464]

36. Ross JS, Wang K, Al-Rohil RN, et al. Advanced urothelial carcinoma: next-generation sequencing reveals diverse genomic alterations and targets of therapy. Mod Pathol. 2014; 27(2):271-80. [PubMed: 23887298]

37. Grivas PD, Day M, Hussain M. Review Article Urothelial carcinomas: a focus on human epidermal receptors signaling. Am J Transl Res. 2011; 3(4):362-373. [PubMed: 21904656]

38. Hussain MH, MacVicar GR, Petrylak DP, et al. Trastuzumab, paclitaxel, carboplatin, and gemcitabine in advanced human epidermal growth factor receptor-2/neu-positive urothelial carcinoma: results of a multicenter phase II National Cancer Institute trial. J Clin Oncol. 2007; 25(16):2218-24. [PubMed: 17538166]

39. Beuzeboc, et al. Trastuzumab (T) combined with standard chemotherapy in HER+ metastatic bladder cancer (BC) patients: Interim safety results of a prospective randomized phase II study. 25(18 Supplement):15565. ASCO Meeting Abstracts [Internet]. Available from: http:// meeting.ascopubs.org/cgi/content/abstract/25/18_supp1/15565.

40. DN24-02 as Adjuvant Therapy in Subjects With High Risk HER2+ Urothelial Carcinoma - Full Text View - ClinicalTrials.gov [Internet]. Available from: http://clinicaltrials.gov/show/ NCT01353222

41. Preliminary safety, product parameters, and immune response assessments from a phase II randomized, open-label trial of DN24-02, an autologous cellular immunotherapy (ACI), in patients (pts) with surgically resected HER2+ urothelial cancer (UC) at high ri [Internet]. Available from: http://meetinglibrary.asco.org/content/114690-132

42. A Double Blind Randomised Study of Lapatinib and Placebo in Metastatic TCC of the Urothelium - Full Text View - ClinicalTrials.gov [Internet]. Available from: http://clinicaltrials.gov/show/ NCT00949455

43**. Ross JS, Wang K, Gay LM, et al. A high frequency of activating extracellular domain ERBB2 (HER2) mutation in micropapillary urothelial carcinoma. Clin Cancer Res. 2014; 20(1):68-75. **Paper demonstrating the signficance of HER2 mutations in UC. [PubMed: 24192927]

44. Ali SM, Alpaugh RK, Downing SR, et al. Response of an ERBB2-Mutated Inflammatory Breast Carcinoma to Human Epidermal Growth Factor Receptor 2-Targeted Therapy. J Clin Oncol. 2014:1-4.

45. An Open-label Phase 2 Study of Neratinib in Patients With Solid Tumors With Somatic Human Epidermal Growth Factor Receptor (EGFR, HER2, HER3) Mutations or EGFR Gene Amplification - Full Text View - ClinicalTrials.gov [Internet]. Available from: http:// clinicaltrials.gov/ct2/show/NCT01953926

46. Normanno N, De Luca A, Bianco C, et al. Epidermal growth factor receptor (EGFR) signaling in cancer. Gene. 2006; 366(1):2-16. [PubMed: 16377102]

47**. Hanahan D, Weinberg RA. The Hallmarks of Cancer. Cell. 2000; 100:57-70. ** Landmark paper in cancer pathogenesis. [PubMed: 10647931]

48. Fiorentino M, Giunchi F, Altimari A, et al. Learning From Errors: Response to Gefitinib in Kidney Urothelial Carcinoma With EGFR Mutations. Oncologist. 2014; 19(4):430. [PubMed: 24652385]

49. Petrylak DP, Tangen CM, Van Veldhuizen PJ, et al. Results of the Southwest Oncology Group phase II evaluation (study S0031) of ZD1839 for advanced transitional cell carcinoma of the urothelium. BJU Int. 2010; 105(3):317-21. [PubMed: 19888985]

50. Wong YN, Litwin S, Vaughn D, et al. Phase II trial of cetuximab with or without paclitaxel in patients with advanced urothelial tract carcinoma. J Clin Oncol. 2012; 30(28):3545-51. [PubMed: 22927525]

51. Philips GK, Halabi S, Sanford BL, et al. A phase II trial of cisplatin (C), gemcitabine (G) and gefitinib for advanced urothelial tract carcinoma: results of Cancer and Leukemia Group B (CALGB) 90102. Ann Oncol. 2009; 20(6):1074-9. [PubMed: 19168670]

52. HDAC inhibitors reverse epithelial-to-mesenchymal transition (EMT) and restore sensitivity to EGFR inhibitors in urothelial carcinoma cells. 2009 Genitourinary Cancers Symposium. Abstracts | Meeting Library [Internet]. Available from: http://meetinglibrary.asco.org/content/20366-64 
53. Iyer G, Milowsky MI. Fibroblast growth factor receptor-3 in urothelial tumorigenesis. Urol Oncol. 2013; 31(3):303-11. [PubMed: 22285006]

54. Billerey C, Chopin D, Bralet M, et al. Frequent FGFR3 Mutations in Pappilary Non-Invasive Bladder (pTa) Tumors. Am J Pathol. 2001; 158(6):1955-1959. [PubMed: 11395371]

55. Van Rhijn BWG, van der Kwast TH, Liu L, et al. The FGFR3 mutation is related to favorable pT1 bladder cancer. J Urol. 2012; 187(1):310-4. [PubMed: 22099989]

56. Iyer G, Al-Ahmadie H, Schultz N, et al. Prevalence and Co-Occurrence of Actionable Genomic Alterations in High-Grade Bladder Cancer. J Clin Oncol. 2013

57. Williams SV, Hurst CD, Knowles MA. Oncogenic FGFR3 gene fusions in bladder cancer. Hum Mol Genet. 2013; 22(4):795-803. [PubMed: 23175443]

58. Di Martino E, Tomlinson DC, Knowles MA. A Decade of FGF Receptor Research in Bladder Cancer: Past, Present, and Future Challenges. Adv Urol. 2012; 2012:429213. [PubMed: 22899908]

59. Lamont FR, Tomlinson DC, Cooper PA, et al. Small molecule FGF receptor inhibitors block FGFR-dependent urothelial carcinoma growth in vitro and in vivo. Br J Cancer. 2011; 104(1):7582. [PubMed: 21119661]

60. Dovitinib in BCG Refractory Urothelial Carcinoma With FGFR3 Mutations or Over-expression Full Text View - ClinicalTrials.gov [Internet]. Available from: http://clinicaltrials.gov/show/ NCT01732107

61. Final results of a multicenter, open-label phase II trial of dovitinib (TKI258) in patients with advanced urothelial carcinoma with either mutated or nonmutated FGFR3. 2013 Genitourinary Cancers Symposium. Abstracts | Meeting Library [Internet]. Available from: http:// meetinglibrary.asco.org/content/106458-134

62. Phase I study of BGJ398, a selective pan-FGFR inhibitor in genetically preselected advanced solid tumors [Internet]. Available from: http://www.abstractsonline.com/Plan/ViewAbstract.aspx? $\mathrm{mID}=3404 \& \mathrm{sKey}=\mathrm{a} 56 \mathrm{e} 6 \mathrm{bb} 6-7 \mathrm{ce} 7-4 \mathrm{f} 58-89 \mathrm{a} 7-\mathrm{f} 118 \mathrm{~d} 4 \mathrm{efb} 710 \& \mathrm{cKey}=25 \mathrm{~b} 2209 \mathrm{a}-5959-45 \mathrm{f} 1-$ a777-4414cb7cdf21\&mKey=6ffe1446-a164-476a-92e7-c26446874d93

63. First in human study of JNJ-42756493, a potent pan fibroblast growth factor receptor (FGFR) inhibitor in patients with advanced solid tumors [Internet]. Available from: http:// www.abstractsonline.com/Plan/ViewAbstract.aspx?mID=3404\&sKey=a56e6bb6-7ce7-4f58-89a7$\mathrm{f} 118 \mathrm{~d} 4 \mathrm{efb} 710 \& \mathrm{cKey}=\mathrm{ad} 57 \mathrm{afb} 0-3 \mathrm{~d} 24-44 \mathrm{fc}-8 \mathrm{ee} 8-\mathrm{ae} 21 \mathrm{e} 404 \mathrm{~d} 85 \mathrm{a} \& \mathrm{mKey}=6 \mathrm{ffe} 1446-$ a164-476a-92e7-c26446874d93

64. Garraway LA, Jänne PA. Circumventing cancer drug resistance in the era of personalized medicine. Cancer Discov. 2012; 2(3):214-26. [PubMed: 22585993]

65. Herrera-Abreu MT, Pearson A, Campbell J, et al. Parallel RNA interference screens identify EGFR activation as an escape mechanism in FGFR3-mutant cancer. Cancer Discov. 2013; 3(9):1058-71. [PubMed: 23744832]

66. A phase II study of cabozantinib in patients (pts) with relapsed or refractory metastatic urothelial carcinoma (mUC). 2014 Genitourinary Cancers Symposium. Abstracts | Meeting Library [Internet]. Available from: http://meetinglibrary.asco.org/content/124412-142

67. Kamada M, So A, Muramaki M, et al. Hsp27 knockdown using nucleotide-based therapies inhibit tumor growth and enhance chemotherapy in human bladder cancer cells. Mol Cancer Ther. 2007; 6(1):299-308. [PubMed: 17218637]

68. A Phase 2 Study Comparing Chemotherapy in Combination With OGX-427 or Placebo in Patients With Bladder Cancer - Full Text View - ClinicalTrials.gov [Internet]. Available from: http:// clinicaltrials.gov/show/NCT01454089

69. Phase 2 Study of Docetaxel +/- OGX-427 in Patients With Relapsed or Refractory Metastatic Bladder Cancer - Full Text View - ClinicalTrials.gov [Internet]. Available from: http:// clinicaltrials.gov/show/NCT01780545

70. Garg M, Kanojia D, Seth A, et al. Heat-shock protein 70-2 (HSP70-2) expression in bladder urothelial carcinoma is associated with tumour progression and promotes migration and invasion. Eur J Cancer. 2010; 46(1):207-15. [PubMed: 19914824]

71. Vignali M, Hassan AH, Neely KE, Workman JL. ATP-Dependent Chromatin-Remodeling Complexes. Mol Cell Biol. 2000; 20(6):1899-910. [PubMed: 10688638] 
72. Gui Y, Guo G, Huang Y, et al. Frequent mutations of chromatin remodeling genes in transitional cell carcinoma of the bladder. Nat Genet. 2011; 43(9):875-8. [PubMed: 21822268]

73. Decitabine in Treating Patients With Advanced Solid Tumors - Full Text View - ClinicalTrials.gov [Internet]. Available from: http://clinicaltrials.gov/ct2/show/NCT00030615

74. Niegisch G, Knievel J, Koch A, et al. Changes in histone deacetylase (HDAC) expression patterns and activity of HDAC inhibitors in urothelial cancers. Urol Oncol. 2013; 31(8):1770-9. [PubMed: 22944197]

75. Kelly WK, Richon VM, O'Connor O, et al. Phase I clinical trial of histone deacetylase inhibitor: suberoylanilide hydroxamic acid administered intravenously. Clin Cancer Res. 2003; 9(10 Pt 1): 3578-88. [PubMed: 14506144]

76. Kelly WK, O'Connor OA, Krug LM, et al. Phase I study of an oral histone deacetylase inhibitor, suberoylanilide hydroxamic acid, in patients with advanced cancer. J Clin Oncol. 2005; 23(17): 3923-31. [PubMed: 15897550]

77. Phase II study of vorinostat (Suberoylanilide Hydroxamic Acid, SAHA) in patients with advanced transitional cell urothelial cancer (TCC) after platinum-based therapy--California Cancer Consortium/University of Pittsburgh NCI/CTEP-sponsored trial. Cheung [Internet]. Available from: http://meeting.ascopubs.org/cgi/content/abstract/26/15_suppl/16058

78. Rosik L, Niegisch G, Fischer U, et al. Limited efficacy of specific HDAC6 inhibition in urothelial cancer cells. Cancer Biol Ther. 2014; 15(6):1. [PubMed: 24309512]

79**. Hanahan D, Weinberg RA. Hallmarks of cancer: the next generation. Cell. 2011; 144(5):646-74. **Updated version of earlier landmark cancer pathogenesis paper. [PubMed: 21376230]

80. Couzin-Frankel J. Breakthrough of the year 2013. Cancer Immunotherapy. Science (80-. ). 2013; 342(6165):1432-3.

81. Investigational Immunotherapy Granted First Breakthrough Therapy Designation in Bladder Cancer [Internet]. OncLive. 2014. Available from: http://www.onclive.com/conference-coverage/ asco-2014/Investigational-Immunotherapy-Granted-First-Breakthrough-Therapy-Designation-inBladder-Cancer

82. Bladder Cancer Treatment (PDQ $\left.{ }^{\circledR}\right)$ - National Cancer Institute [Internet]. Available from: http:// www.cancer.gov/cancertopics/pdq/treatment/bladder/HealthProfessional/page1/ AllPages\&num;Section_323

83. Lamm D, Brausi M, O'Donnell MA, Witjes JA. Interferon alfa in the treatment paradigm for nonmuscle-invasive bladder cancer. Urol Oncol. 2014; 32(1):35.e21-30. [PubMed: 23628309]

84. Carthon BC, Wolchok JD, Yuan J, et al. Preoperative CTLA-4 blockade: tolerability and immune monitoring in the setting of a presurgical clinical trial. Clin Cancer Res. 2010; 16(10):2861-71. [PubMed: 20460488]

85. First-Line Gemcitabine, Cisplatin + Ipilimumab for Metastatic Urothelial Carcinoma - Full Text View - ClinicalTrials.gov [Internet]. Available from: http://clinicaltrials.gov/show/NCT01524991

86. Inhibition of PD-L1 by MPDL3280A and clinical activity in pts with metastatic urothelial bladder cancer (UBC). 2014 ASCO Annual Meeting. Abstracts [Internet]. Available from: http:// abstracts.asco.org/144/AbstView_144_128960.html

87. A Study of MPDL3280A in Patients With Locally Advanced or Metastatic Urothelial Bladder Cancer - Full Text View - ClinicalTrials.gov [Internet]. Available from: http:// clinicaltrials.gov/ct2/show/NCT02108652?term=anti+PD1+urothelial\&rank=1

88. Oral Lenalidomide and Intravesical BCG for Therapy of Bladder Cancer - Full Text View ClinicalTrials.gov [Internet]. Available from: http://clinicaltrials.gov/show/NCT01373294

89**. Zamble DB, Lippard SJ. Cisplatin and DNA repair in cancer chemotherapy. Trends Biochem Sci. 1995; 20(10):435-9. ** Seminal work explaining mechanism of cisplatin activity in cancers. [PubMed: 8533159]

90. Konstantakou EG, Voutsinas GE, Karkoulis PK. Human bladder cancer cells undergo cisplatininduced apoptosis that is associated with p53-dependent and p53-independent responses. 2009:401-416.

91. Walker MC, Povey S, Parrington JM, et al. Development and characterization of cisplatin-resistant human testicular and bladder tumour cell lines. Eur J Cancer. 1990; 26(6):742-7. [PubMed: 2144165] 
92. Drayton RM, Catto J. Molecular mechanisms of cisplatin resistance in bladder cancer. Expert Opin Anticancer Ther. 2012; 12(2):271-281.

93. Sandlow J, Cohen MB, Robinson RA, et al. DNA ploidy and P-glycoprotein expression as predictive factors of response to neoadjuvant chemotherapy for invasive bladder cancer. Urology. 1994; 43(6):787-91. [PubMed: 7910989]

94. Bahnson RR, Becich M, Ernstoff MS, et al. Absence of immunohistochemical metallothionein staining in bladder tumor specimens predicts response to neoadjuvant cisplatin, methotrexate and vinblastine chemotherapy. J Urol. 1994; 152(6 Pt 2):2272-5. [PubMed: 7966723]

95. Kimiya K, Naito S, Soejima T, et al. Establishment and characterization of doxorubicin-resistant human bladder cancer cell line, KK47/ADM. J Urol. 1992; 148(2 Pt 1):441-5. [PubMed: 1353119]

96. Kim WJ, Kakehi Y, Hirai M, et al. Multidrug resistance-associated protein- mediated multidrug resistance modulated by cyclosporin A in a human bladder cancer cell line. Jpn J Cancer Res. 1995; 86(10):969-77. [PubMed: 7493917]

97. Clifford SC, Neal DE, Lunec J. Alterations in expression of the multidrug resistance-associated protein (MRP) gene in high-grade transitional cell carcinoma of the bladder. Br J Cancer. 1996; 73(5):659-66. [PubMed: 8605104]

98. Taniguchi K, Wada M, Kohno K, Kawakami M. Cancer Cell Lines with Decreased Drug Accumulation AHuman Canalicular Multispecific Organic Anion Transporter (cMOAT) Gene. Cancer Res. 1996:4124-4129. [PubMed: 8797578]

99. Tada YT, Wada MW, Igita TM, et al. Increasd expression of Multidrug Resisitance-Associated Proteins in bladder cancer during clinical course and drug resistance to Doxorubicin. Int J Cancer. 2002; 635:630-635. [PubMed: 11920626]

100. Masters JRW, Thomas R, Ha AG, et al. Sensitivity of Testis Tumour Cells to Chemotherapeutic Drugs: Role of Detoxiwg Pathways. Eur J Cancer. 1996; 32A(7):1248-53. [PubMed: 8758261]

101. Xu BH, Gupta V, Singh SV. Characterization of a human bladder cancer cell line selected for resistance to mitomycin. C Int J Cancer. 1994; 58(5):686-92.

102. Yokomizo A, Ono M, Nanri H, Mitomycin C. Cellular Levels of Thioredoxin Associated with Drug Sensitivity to Cisplatin, Mitomycin C, Doxorubicin, and Etoposide Advances in Brief Cellular Levels of Thioredoxin Associated with Drug Sensitivity to Cisplatin. Cancer Res. 1995:4293-4296. [PubMed: 7671238]

103. Van Allen EM, Mouw KW, Kim P, et al. Somatic ERCC2 Mutations Correlate with Cisplatin Sensitivity in Muscle-Invasive Urothelial Carcinoma. Cancer Discov. 2014; 4(10):1140-53. [PubMed: 25096233]

104. Clinical Study of Microdosing Carboplatin in Lung or Bladder Cancer - Tabular View ClinicalTrials.gov [Internet]. Available from: http://clinicaltrials.gov/ct2/show/record/ NCT01261299

105. Cho HJ, Kim JK, Kim KD, et al. Upregulation of Bcl-2 is associated with cisplatin-resistance via inhibition of Bax translocation in human bladder cancer cells. Cancer Lett. 2006; 237(1):56-66. [PubMed: 16009487]

106. Bolenz C, Becker A, Trojan L, et al. Optimizing chemotherapy for transitional cell carcinoma by application of bcl-2 and bcl-xL antisense oligodeoxynucleotides. Urol Oncol. 2007; 25(6):47682. [PubMed: 18047955]

107. Miyake H, Hanada N, Nakamura H, et al. Overexpression of Bcl-2 in bladder cancer cells inhibits apoptosis induced by cisplatin and adenoviral-mediated p53 gene transfer. Oncogene. 1998; 16(7):933-43. [PubMed: 9484785]

108. Yoon CY, Park MJ, Lee JS, et al. The histone deacetylase inhibitor trichostatin A synergistically resensitizes a cisplatin resistant human bladder cancer cell line. J Urol. 2011; 185(3):1102-11. [PubMed: 21255805] 

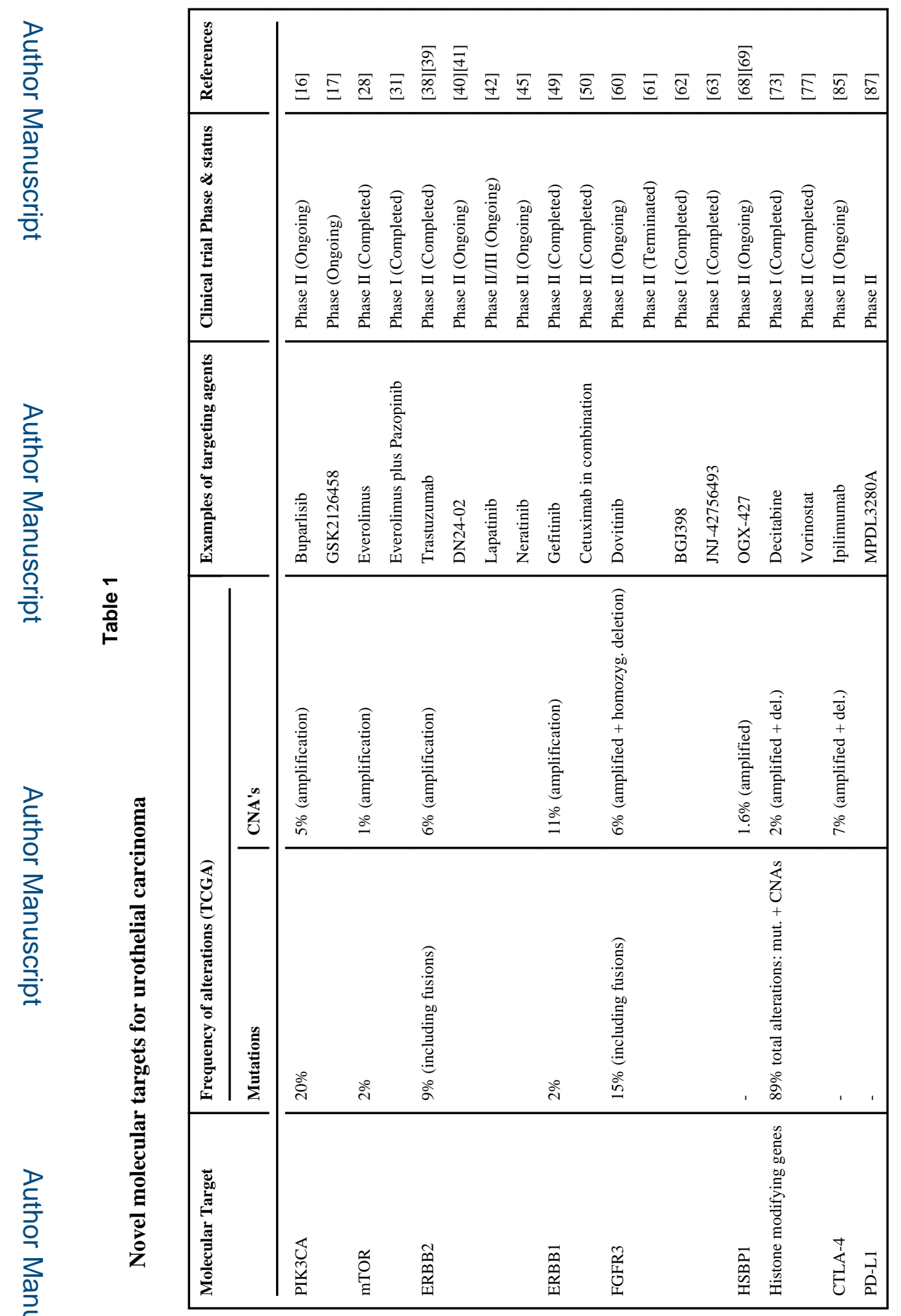

Expert Opin Ther Targets. Author manuscript; available in PMC 2016 April 01. 